\title{
CONTROLE DEPENDENTE DE PARÂMETROS PARA UMA CLASSE DE SISTEMAS NÃO-LINEARES INCERTOS COM ATUADORES SATURANTES
}

\author{
Jones Corso* \\ jonesedas.ufsc.br
}

Ubirajara F. Moreno*

moreno@das.ufsc.br
Eugênio B. Castelan*
eugenio@das.ufsc.br

Edson R. De Pieri*

edsonedas.ufsc.br

${ }^{*}$ Grupo de Controle de Sistemas Mecatrônicos (CSM), DAS/UFSC, Florianópolis (S.C.), Brasil

\section{ABSTRACT}

This paper presents some results on the stabilization by a parameter dependent control law of a class of nonlinear discrete-time systems with some time-varying parameters and under control saturations. The studied control law consists of the feedback of both the states and of the nonlinearity present in the dynamics of the controlled system. Saturations are taken into account by modeling the nonlinear saturated system through a deadzone nonlinearity satisfying a modified sector condition. Thus, LMI stabilization conditions are proposed, which can be cast into convex programming problems to synthesizing the gain matrices that allows to construct the parameter dependent control law. The presented numerical example shows some performance issues and compares the proposed parameter dependent control law with a fixed parameters one.

KEYWORDS: Nonlinear systems, Saturations, Parameter dependent Lyapunov functions, Parameter dependent control, LMIs, Robustness.

\section{RESUMO}

Este artigo apresenta alguns resultados sobre estabilização via uma lei de controle dependente de parâmetros de uma classe de sistemas não-lineares em tempo discreto com parâmetros variantes e sob saturação de controle. A lei de

Artigo submetido em 03/10/2008 (Id.: 00901)

Revisado em 15/11/2008, 19/01/2009

Aceito sob recomendação do Editor Associado Prof. Takashi Yoneyama controle consiste na realimentação dos estados e da nãolinearidade presente na dinâmica da planta, assumindo-se que o vetor de parâmetros é mensurável em tempo real. Para modelar o sistema não-linear sob saturação, utiliza-se uma não-linearidade de tipo zona-morta satisfazendo uma condição de setor modificada. Assim como para sistemas precisamente conhecidos, são propostas condições de estabilização via LMIs que podem ser utilizados em problemas de programação convexa para a síntese da lei de controle. O exemplo numérico apresentado mostra o desempenho da lei de controle dependente de parâmetros e faz uma comparação com uma a parâmetros fixos.

PALAVRAS-CHAVE: Sistemas não-lineares, Saturações, Função de Lyapunov dependente de parâmetros, Controle dependente de parâmetros, LMIs, Robustez.

\section{INTRODUÇÃO}

A consideração de não-linearidades inerentes à dinâmica de plantas a controlar e de limites físicos de atuadores e sensores é um tópico importante de pesquisa em controle devido ao interesse prático e aos desafios em termos teóricos. Neste sentido, problemas de controle não-linear receberam especial atenção com a proposta de novas ferramentas de projeto, através de procedimentos construtivos aplicáveis a classes de sistemas não-lineares (Kokotovic and Arcak, 2001; Arcak et al., 2003; Arcak and Kokotovic, 2001) e, em particular, considerando a saturação de atuadores, como por exemplo em (Tarbouriech et al., 2004; Gomes da Silva Jr. et al., 2001; Hu et al., 2002; Castelan et al., 2006) no caso 
de sistemas lineares precisamente conhecidos, (Montagner, Peres, Queinnec and Tarbouriech, 2006) no caso de sistemas lineares incertos e (Ghiggi and Gomes da Silva Jr., 2006) no caso de sistemas com atraso no tempo.

Na linha dos trabalhos que tratam a saturação de atuadores, encontramos em (Castelan et al., 2005; Castelan et al., 2006; Castelan et al., 2007; Castelan et al., 2008) resultados relacionados a uma classe de sistemas não-lineares que podem ser representados pela interconexão de um sistema linear e de uma não-linearidade associada à dinâmica da planta e pertencente a um setor de tipo cone limitado. Neste trabalho, pretende-se estender os resultados propostos em (Castelan et al., 2007) para tratar sistemas com parâmetros variantes no tempo, considerando uma lei de controle dependente de parâmetros. A lei de controle sob saturação consiste na realimentação dos estados e da não-linearidade do sistema a controlar, e requer que o parâmetro variante seja mensurável em tempo real. Desta forma, o problema formulado e a técnica proposta também permitem considerar o controle de sistemas em que parte das não-linearidades pode ser representada como dependente de parâmetros variantes no tempo (veja (Ghaoui and Scorletti, 1996; Ghaoui and Niculescu, 2000)) e/ou não-linearidades de tipo setor.

Como ferramentas para tratamento do problema de controle utilizamos uma Função de Lyapunov Dependente de Parâmetros (FLDP), a qual é associado um Conjunto de tipo Lyapunov (CL), e uma condição de setor modificada para levar em conta a não-linearidade de saturação. Assim, para assegurar a convergência das trajetórias do sistema em malha fechada para a origem, com um certo desempenho temporal, propomos condições do tipo LMI que garantem a propriedade de $\lambda$-contratividade (Milani and Coelho, 2002) de um conjunto de condições iniciais. Esta propriedade é assegurada de forma robusta em relação as possíveis variações paramétricas e para toda não-linearidade pertencente ao setor sob consideração. O conjunto de condições iniciais é formado pela interseção de elipses associadas a cada um dos vértices do politopo de matrizes que descreve o sistema não-linear a controlar. Os resultados numéricos relatados permitem verificar a eficiência da estratégia de controle sob saturação, bem como evidenciar alguns compromissos existentes entre robustez, desempenho e tamanho do conjunto de condições iniciais obtido via o problema de otimização convexa proposto para a síntese das matrizes do controlador variante.

O artigo é organizado da seguinte forma: na Seção 2, o problema de síntese sob saturação é apresentado; na Seção 3, apresentamos alguns resultados preliminares e definições e, na Seção 4, as condições de estabilização local são deduzidas e um problema de programação convexa é formulado para obter os parâmetros do controlador; um exemplo numérico e comentários são apresentados na Seção 5. E ao encerrar o artigo apresentamos algumas observações finais.

Notações. Relativo à matriz $A \in \Re^{n \times n}, A^{\prime}$ denota a transposta, e $A_{(i)}, i=1, \ldots n$, denota a $i$-ésima coluna. Se $A=A^{\prime} \in \Re^{n \times n}$, então $A<0(A \leq 0)$ significa que $A$ é definida (semi) negativa. Os componentes de qualquer vetor $x \in \Re^{n}$ são representados por $x_{(i)}, \forall i=1, \ldots n$. Desigualdades entre vetores são consideradas por componentes: $x \leq 0$ significa que $x_{(i)} \leq 0$ e $x \leq y$ implica que $x_{(i)}-y_{(i)} \leq 0$. $I_{n}$ denota uma matriz identidade $n \times n$.

\section{APRESENTAÇÃO DO PROBLEMA}

Considere um sistema não-linear em tempo discreto com parâmetros variantes no tempo, representado por:

$$
\begin{aligned}
x_{k+1} & =A\left(\xi_{k}\right) x_{k}+G\left(\xi_{k}\right) \varphi\left(z_{k}\right)+B\left(\xi_{k}\right) \operatorname{sat}\left(u_{k}\right) \\
z_{k} & =L\left(\xi_{k}\right) x_{k}
\end{aligned}
$$

em que $x_{k} \in \Re^{n}, u_{k} \in \Re^{m}, z_{k} \in \Re^{p}, \varphi():. \Re^{p} \rightarrow \Re^{p}$ e $\xi_{k} \subset \Re^{N}$ é um vetor de parâmetros mensurável, variante no tempo e limitado no simplex unitário: $\Xi=\left\{\xi_{k} \in\right.$ $\left.\Re^{N} ; \sum_{i=1}^{N} \xi_{k(i)}=1, \xi_{k(i)} \geq 0, i=1, \ldots, N\right\}$. A estrutura das matrizes do sistema tem a forma seguinte:

$$
\begin{aligned}
& {\left[\begin{array}{llll}
A\left(\xi_{k}\right) & B\left(\xi_{k}\right) & G\left(\xi_{k}\right) & L\left(\xi_{k}\right)
\end{array}\right]=} \\
& \sum_{i=1}^{N} \xi_{k(i)}\left[\begin{array}{llll}
A_{i} & B_{i} & G_{i} & L_{i}
\end{array}\right]
\end{aligned}
$$

A não-linearidade $\varphi($.$) verifica uma condição de setor de$ tipo cone limitado, $\varphi(.) \in[0, \Omega], \forall z \in \Re^{p}$ (Khalil, 2002), i.e.: existe uma matriz simétrica definida positiva $\Omega=\Omega^{\prime} \in$ $\Re^{p \times p}$ tal que

$$
\varphi^{\prime}\left(z_{k}\right) \Delta\left[\varphi\left(z_{k}\right)-\Omega z_{k}\right] \leq 0, \quad \begin{aligned}
& \forall z_{k} \in \Re^{p} \\
& \varphi(0)=0
\end{aligned}
$$

no qual $\Delta \in \Re^{p \times p}$ é uma matriz diagonal qualquer:

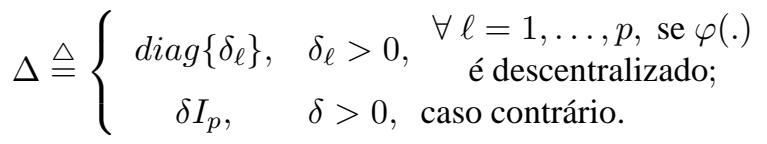

Por definição, a não-linearidade $\varphi($.$) satisfaz globalmente a$ condição de setor (3). Entretanto, o caso no qual esta nãolinearidade é válida localmente também podem ser tratado. Note que a matriz $\Omega$ é um dado do projeto e considerada como conhecida na sequência. Por outro lado, a partir da definição de $\Delta$, vemos que (3) é verificada se, e somente se, a condição de setor clássica $\varphi^{\prime}\left(z_{k}\right)\left[\varphi\left(z_{k}\right)-\Omega z_{k}\right] \leq 0$ é também verificada. Deste modo, $\Delta$ representa um grau de liberdade (uma variável de decisão a ser utilizada posteriormente) (Castelan et al., 2007). 
As entradas de controle são limitadas em amplitude e o modelo da função saturação considerado é:

$$
\operatorname{sat}\left(u_{(\ell)}(t)\right)=\operatorname{sign}\left(u_{(\ell)}(t)\right) \min \left(\rho_{(\ell)},\left|u_{(\ell)}(t)\right|\right)
$$

$\forall \ell=1, \ldots, m$, em que $\rho_{(\ell)}>0$ denota o limite simétrico da amplitude relativo ao $l$-ésimo controle.

No decorrer deste trabalho, assumimos que o vetor de parâmetros, $\xi_{k}$, é mensurável em tempo real. Então, a lei de controle dependente de parâmetros seguinte é considerada:

$$
u_{k}=K\left(\xi_{k}\right) x_{k}+\Gamma\left(\xi_{k}\right) \varphi\left(z_{k}\right)
$$

A estrutura das matrizes da lei de controle é:

$$
\left[\begin{array}{ll}
K\left(\xi_{k}\right) & \Gamma\left(\xi_{k}\right)
\end{array}\right]=\sum_{i=1}^{N} \xi_{k(i)}\left[\begin{array}{ll}
K_{i} & \Gamma_{i}
\end{array}\right]
$$

na qual $K\left(\xi_{k}\right)$ é uma matriz de realimentação de estado, com $K_{i} \in \Re^{m \times n}$, e $\Gamma\left(\xi_{k}\right)$ é uma realimentação associada à nãolinearidade $\varphi(),. \operatorname{com} \Gamma_{i} \in \Re^{m \times p}$.

Temos, assim, o sistema em malha fechada:

$$
x_{k+1}=\begin{gathered}
A\left(\xi_{k}\right) x_{k}+G\left(\xi_{k}\right) \varphi\left(z_{k}\right)+ \\
B\left(\xi_{k}\right) \operatorname{sat}\left(K\left(\xi_{k}\right) x_{k}+\Gamma\left(\xi_{k}\right) \varphi\left(z_{k}\right)\right)
\end{gathered}
$$

Problema 1 (Estabilização com controle dependente de parâmetros sob atuador saturante) Determine as matrizes de ganho de realimentação $K_{i}$ e $\Gamma_{i}$ que formam a lei de controle dependente de parâmetros (6) e (7), e uma região $\mathcal{S}_{0} \subseteq \Re^{n}$, tão grande quanto possível, tais que para qualquer condição inicial $x_{0} \in \mathcal{S}_{0}$ a origem do sistema em malha fechada (8) é assintoticamente estável para qualquer $\varphi($.$) verificando a$ condição de setor (3).

Note-se que (6) tem a mesma estrutura das leis de controle com ganhos fixos adotada em (Castelan et al., 2006; Castelan et al., 2008), no caso de sistemas precisamente conhecidos, e (Castelan et al., 2007), no caso de sistemas incertos. Como nos trabalhos anteriores, a aplicação da lei de controle (6) requer o conhecimento ou a disponibilidade da não-linearidade $\varphi($.$) como um sinal (Arcak et al., 2003).$

\section{RESULTADOS PRELIMINARES}

Seja a não-linearidade genérica de tipo zona-morta, dada por

$$
\Psi\left(u_{k}\right)=u_{k}-\operatorname{sat}\left(u_{k}\right) .
$$

Considerando $u_{k}$ dado por (6), podemos reescrever o sistema em malha fechada (8) sob a forma

$$
x_{k+1}=A_{c l}\left(\xi_{k}\right) x_{k}+G_{c l}\left(\xi_{k}\right) \varphi\left(z_{k}\right)-B\left(\xi_{k}\right) \Psi\left(u_{k}\right)
$$

na qual $\left[\begin{array}{lll}A_{c l}\left(\xi_{k}\right) & G_{c l}\left(\xi_{k}\right) & B\left(\xi_{k}\right)\end{array}\right]=\sum_{i=1}^{N} \xi_{k(i)}$

$$
\left[A_{i}+B_{i} \sum_{j=1}^{N} \xi_{k(j)} K_{j} \quad G_{i}+B_{i} \sum_{j=1}^{N} \xi_{k(j)} \Gamma_{j} \quad B_{i}\right] .
$$

Seja o conjunto poliedral:

$$
S(\rho)=\left\{u_{k} \in \Re^{m}, w_{k} \in \Re^{m} ;-\rho \leq u_{k}-w_{k} \leq \rho\right\}
$$

O Lema seguinte permite considerar a zona-morta como uma não-linearidade que pertence a uma condição de setor modificada (Gomes da Silva Jr. and Tarbouriech, 2005).

Lema 1 Se $u_{k}$ e $w_{k}$ são elementos de $S(\rho)$, então a nãolinearidade $\Psi\left(u_{k}\right)$ satisfaz a desigualdade

$$
\Psi\left(u_{k}\right)^{\prime} T\left(\xi_{k}\right)\left(\Psi\left(u_{k}\right)-w_{k}\right) \leq 0
$$

para qualquer matriz diagonal e positiva $T($.$) de dimensão$ $m \times m$.

Demonstração: Segue as mesmas linhas de (Tarbouriech et al., 2004) e (Montagner, Peres, Tarbouriech and Queinnec, 2006).

Para obter condições de estabilização sob a forma de LMIs, nós consideramos $u_{k}$ dado por (6) e o vetor $w_{k}$ será estruturado de forma similar à $u_{k}$, como em (Castelan et al., 2006; Castelan et al., 2007):

$$
w_{k}=E_{1}\left(\xi_{k}\right) x_{k}+E_{2}\left(\xi_{k}\right) \varphi\left(z_{k}\right)
$$

com

$$
\begin{aligned}
& {\left[\begin{array}{ll}
E_{1}\left(\xi_{k}\right) & E_{2}\left(\xi_{k}\right)
\end{array}\right]=\sum_{i=1}^{N} \xi_{k(i)}\left[\begin{array}{ll}
E_{1 i} & E_{2 i}
\end{array}\right],} \\
& E_{1} \in \Re^{m \times n} \text { e } E_{2} \in \Re^{m \times p} .
\end{aligned}
$$

Observação 1 Uma outra escolha possível para o vetor $w_{k}$ seria sob a forma:

$$
w_{k}=\Lambda K\left(\xi_{k}\right) x_{k}+\Lambda \Gamma\left(\xi_{k}\right) \varphi\left(z_{k}\right)
$$

$\operatorname{com} \Lambda=\operatorname{diag}\left(\lambda_{\ell}\right)$ e $0<\lambda_{\ell} \leq 1, \forall \ell=1, \ldots, m$. Note, entretanto, que este tipo de escolha clássica (veja, por exemplo, (Khalil, 2002)) é um caso particular de (13) e, além disso, implica na utilização de técnicas de otimização para a solução de BMIs devido à não-linearidade intrínsica à busca conjunta dos coeficientes $\lambda_{\ell}$ e das matrizes $K_{i}$ e $\Gamma_{i}$ em (6) e (7).

Considere também uma Função de Lyapunov Dependente de Parâmetros (FLDP), $V\left(x_{k}, \xi_{k}\right): \Re^{n} \times \Xi \longrightarrow \Re^{+}$. Um Conjunto de tipo Lyapunov (CL) associado a $V\left(x_{k}, \xi_{k}\right)$ é dado por

$$
L_{V} \triangleq\left\{x_{k} \in \Re^{n} ; V\left(x_{k}, \xi_{k}\right) \leq 1, \forall \xi_{k} \in \Xi\right\}
$$


A noção de conjunto contrativo é básica para determinar regiões de estabilidade assintótica para o sistema em malha fechada (10) (Milani and Coelho, 2002). A definição seguinte de $\lambda$-contratividade é adaptada para considerar incertezas nos parâmetros e a caracterização da não-linearidade de setor limitado $\varphi($.$) .$

Definição 1 Seja $\lambda \in(0,1]$. O conjunto $L_{V}$ é robustamente absolutamente $\lambda$-contrativo (com respeito as trajetórias do sistema (10)), se

$$
\begin{gathered}
\Delta V(x, \xi) \triangleq V\left(x_{k+1}, \xi_{k+1}\right)-\lambda V\left(x_{k}, \xi_{k}\right)<0 \\
\left\{\begin{array}{c}
\forall x_{k} \in L_{V}, \forall \xi_{k} \in \Xi \\
\mathrm{e} \forall \varphi(.) \in[0, \Omega]
\end{array}\right.
\end{gathered}
$$

Observe que se $L_{V}$ satisfaz a Definição 1, então

$$
V\left(x_{k}, \xi_{k}\right)<\lambda V\left(x_{k-1}, \xi_{k-1}\right)<\ldots<\lambda^{k} V\left(x_{0}, \xi_{0}\right) \leq 1 .
$$

Assim, a cada instante de tempo $k>0$, a trajetória do sistema converge assintoticamente para a origem, i.e.: $\lim _{k \rightarrow \infty} x_{k}=0, \forall x_{0} \in L_{V}$.

Na sequência, tomemos a FLDP na forma (Hu and Lin, 2003)

$$
V\left(x_{k}, \xi_{k}\right)=x_{k}^{\prime} Q^{-1}\left(\xi_{k}\right) x_{k},
$$

em que $Q\left(\xi_{k}\right)=\sum_{i=1}^{N} \xi_{k(i)} Q_{i}, \quad Q_{i}=Q_{i}^{\prime}>0$.

Lema 2 Considere os conjuntos elipsoidais:

$$
\mathcal{E}\left(Q_{i}^{-1}\right)=\left\{x \in \Re^{n} ; x^{\prime} Q_{i}^{-1} x \leq 1\right\}, \forall i=1, \ldots, N .
$$

Então, o CL obtido de (15) e (17) é a interseção dos conjuntos elipsoidais (18):

$$
\mathcal{E}\left(Q^{-1}\left(\xi_{k}\right)\right) \triangleq \bigcap_{\xi_{k} \in \Xi}\left\{\mathcal{E}\left(Q^{-1}\left(\xi_{k}\right)\right)\right\}=\bigcap_{i \in\{1, \ldots, N\}} \mathcal{E}\left(Q_{i}^{-1}\right)
$$

Demonstração: Veja Anexo II.

Observamos, a partir do Lema 2, que o conjunto $L_{V}$ considerado é independente do parâmetro variante no tempo $\xi_{k}$, o que permite analisar a contratividade de forma robusta, $\forall \xi_{k} \in \Xi$.

\section{RESULTADOS PRINCIPAIS}

\subsection{Estabilização Robusta}

Visando o projeto de matrizes de realimentação $K_{i}$ e $\Gamma_{i}$, propomos o resultado seguinte:
Proposição 1 Dado $\lambda \in(0,1]$, considere que existem matrizes definidas positiva $Q_{i} \in \Re^{n \times n}$, matrizes diagonais positiva $S_{i} \in \Re^{m \times m}$ e $S_{\Delta} \in \Re^{p \times p}$, e matrizes $Z_{1 i} \in \Re^{m \times n}$, $Z_{2 i} \in \Re^{m \times p}, U \in \Re^{n \times n}, Y_{1 i} \in \Re^{m \times n}$ e $Y_{2 i} \in \Re^{m \times p}$ que verificam:

$$
\begin{gathered}
\mathcal{M}_{j i}^{+}=\left[\begin{array}{cccc}
-Q_{j} & \mathbf{M}_{\mathbf{1 i}} & \mathbf{M}_{\mathbf{2 i}} & -B_{i} S_{i} \\
\star & \lambda \mathbf{M}_{\mathbf{3 i}} & U^{\prime} L_{i}^{\prime} \Omega & Z_{1 i}^{\prime} \\
\star & \star & -2 S_{\Delta} & Z_{2 i}^{\prime} \\
\star & \star & \star & -2 S_{i}
\end{array}\right]<0 \\
\forall j=1, \ldots, N \text { e } \forall i=1, \ldots, N \\
\mathcal{M}_{j i q}^{+}=\left[\begin{array}{cccc}
-2 Q_{j} & \mathbf{M}_{\mathbf{4 i q}} & \mathbf{M}_{\mathbf{5 i q}} & \mathbf{M}_{\mathbf{8 i q}} \\
\star & \lambda \mathbf{M}_{\mathbf{6 i q}} & \mathbf{M}_{\mathbf{7 i q}} & Z_{1 i}^{\prime}+Z_{1 q}^{\prime} \\
\star & \star & -2 S_{\Delta} & Z_{2 i}^{\prime}+Z_{2 q}^{\prime} \\
\star & \star & \star & -2\left(S_{i}+S_{q}\right)
\end{array}\right]<0 \\
\forall j=1, \ldots, N, \forall i=1, \ldots, N-1
\end{gathered}
$$

$$
\begin{gathered}
{\left[\begin{array}{ccc}
-\mathbf{M}_{\mathbf{3 i}} & -U^{\prime} L_{i}^{\prime} \Omega & Y_{1 i(\ell)}^{\prime}-Z_{1 i(\ell)}^{\prime} \\
\star & 2 S_{\Delta} & Y_{2 i(\ell)}^{\prime}-Z_{2 i(\ell)}^{\prime} \\
\star & \star & \rho_{(\ell)}^{2}
\end{array}\right] \geq 0} \\
\forall i=1, \ldots, N e \forall \ell=1, \ldots, m
\end{gathered}
$$

nas quais:

$$
\begin{gathered}
\mathbf{M}_{\mathbf{1 i}}=A_{i} U+B_{i} Y_{1 i}, \\
\mathbf{M}_{\mathbf{2 i}}=G_{i} S_{\Delta}+B_{i} Y_{2 i}, \\
\mathbf{M}_{\mathbf{3 i}}=Q_{i}-U-U^{\prime}, \\
\mathbf{M}_{\mathbf{4 i q}}=\left(A_{i}+A_{q}\right) U+B_{i} Y_{1 q}+B_{q} Y_{1 i}, \\
\mathbf{M}_{\mathbf{5 i q}}=\left(G_{i}+G_{q}\right) S_{\Delta}+B_{i} Y_{2 q}+B_{q} Y_{2 i}, \\
\mathbf{M}_{\mathbf{6 i q}}=Q_{i}+Q_{q}-2 U-2 U^{\prime}, \\
\mathbf{M}_{\mathbf{7 i q}}=U^{\prime}\left(L_{i}^{\prime}+L_{q}^{\prime}\right) \Omega \\
\mathbf{M}_{\mathbf{8 i q}}=-\left(B_{i} S_{q}^{\prime}+B_{q} S_{i}^{\prime}\right) .
\end{gathered}
$$

Então, os ganhos $K_{i}$ e $\Gamma_{i}$ que verificam

$$
K_{i} U=Y_{1 i}, \quad \Gamma_{i} S_{\Delta}=Y_{2 i}
$$

e o conjunto $\mathcal{S}_{0} \triangleq \bigcap_{i}\left\{\mathcal{E}\left(Q_{i}^{-1}\right)\right\}=\mathcal{E}\left(Q^{-1}(\xi)\right)$, são soluções para o Problema 1 para qualquer não-linearidade $\varphi(z)$ satisfazendo a relação (3) $\operatorname{com} \Delta=S_{\Delta}^{-1}$.

Demonstração: De (19), percebemos primeiramente que $U$ e $S_{\Delta}$ são, necessariamente, invertíveis. Assim, podemos definir $E_{1 i} \in \Re^{m \times n}$ e $E_{2 i} \in \Re^{m \times p}$ como segue:

$$
E_{1 i}=Z_{1 i} U^{-1}, E_{2 i}=Z_{2 i} S_{\Delta}^{-1}
$$


A partir de (22) e (23), (21) pode ser reescrita:

$$
\left[\begin{array}{ccc}
-\mathbf{M}_{3 \mathbf{i}} & -U^{\prime} L_{i}^{\prime} \Omega & U^{\prime}\left(K_{i(\ell)}^{\prime}-E_{1 i(\ell)}^{\prime}\right) \\
\star & 2 S_{\Delta} & S_{\Delta}\left(\Gamma_{i(\ell)}^{\prime}-E_{2 i(\ell)}^{\prime}\right) \\
\star & \star & \rho_{(\ell)}^{2}
\end{array}\right] \geq 0
$$

Multiplicando a desigualdade em (24) por $\xi_{i}$, para todo $i=$ $1, \ldots, N$ e somando-as, temos:

$$
\left[\begin{array}{ccc}
-Q(\xi)+U+U^{\prime} & -U^{\prime} L^{\prime}(\xi) \Omega & \mathbf{M}_{\mathbf{1}}(\xi) \\
\star & 2 S_{\Delta} & \mathbf{M}_{2}(\xi) \\
\star & \star & \rho_{(\ell)}^{2}
\end{array}\right] \geq 0
$$

em que:

$$
\begin{gathered}
\mathbf{M}_{\mathbf{1}}(\xi)=U^{\prime}\left(K_{(\ell)}^{\prime}(\xi)-E_{1(\ell)}^{\prime}(\xi)\right) \quad e \\
\mathbf{M}_{\mathbf{2}}(\xi)=S_{\Delta}\left(\Gamma_{(\ell)}^{\prime}(\xi)-E_{2(\ell)}^{\prime}(\xi)\right),
\end{gathered}
$$

nas quais $E_{1}(\xi)$ e $E_{2}(\xi)$ são definidos em (14).

Como $(U-Q(\xi))^{\prime} Q(\xi)^{-1}(U-Q(\xi)) \geq 0 \Longleftrightarrow$ $U^{\prime} Q(\xi)^{-1} U \geq-Q(\xi)+U+U^{\prime}$, obtemos:

$$
\left[\begin{array}{ccc}
Q^{-1}(\xi) & -L^{\prime}(\xi) \Omega S_{\Delta}^{-1} & K_{(\ell)}^{\prime}(\xi)-E_{1(\ell)}^{\prime}(\xi) \\
\star & 2 S_{\Delta}^{-1} & \Gamma_{(\ell)}^{\prime}(\xi)-E_{2(\ell)}^{\prime}(\xi) \\
\star & \star & \rho_{(\ell)}^{2}
\end{array}\right] \geq 0 .
$$

Segue que, para todo e qualquer $\xi \in \Xi$, a relação (21) garante que $\mathcal{E}\left(Q^{-1}(\xi)\right)$ está incluída em $S(\rho)=\left\{x \in \Re^{n}, \varphi \in \Re^{r} ;-\rho \leq\left(K_{a}(\xi)-\right.\right.$ $\left.\left.E_{a}(\xi)\right)\left[\begin{array}{l}x \\ \varphi\end{array}\right] \leq \rho\right\}$, em que $\left[\begin{array}{cc}K_{a}(\xi) & \left.E_{a}(\xi)\right]=\end{array}\right.$ $\left[\begin{array}{ll}K(\xi)-E_{1}(\xi) & \Gamma(\xi)-E_{2}(\xi)\end{array}\right]$. Então, o Lema 1 pode ser aplicado à não-linearidade $\Psi(u)$ considerando o vetor $u$ dado por (6) e escolhendo o vetor $w=E_{1}(\xi) x+E_{2}(\xi) \varphi(z)$, (13), de forma que a não-linearidade $\Psi(u)$ satisfaz a condição de setor (12), $\forall x \in \mathcal{E}\left(Q^{-1}(\xi)\right)=\bigcap_{i} \mathcal{E}\left\{Q_{i}^{-1}\right\}$.

Para assegurar que (16) é verificada, nós consideramos a desigualdade

$$
\begin{gathered}
\Delta V(x, \xi)-2 \varphi(z) S_{\Delta}^{-1}(\varphi(z)-\Omega L(\xi) x) \\
-2 \Psi(u) S(\xi)^{-1}\left(\Psi(u)+E_{1}(\xi) x+E_{2}(\xi) \varphi(z)\right)<0
\end{gathered}
$$

Utilizando $V\left(x_{k}, \xi_{k}\right)$ dada por (17), (25) pode ser reescrita como:

$$
\left[\begin{array}{ccc}
x^{\prime} & \varphi^{\prime} & \Psi^{\prime}
\end{array}\right] \mathcal{M}(\xi)\left[\begin{array}{l}
x \\
\varphi \\
\Psi
\end{array}\right]<0
$$

na qual, utilizando as trocas de variáveis representadas por (22) e (23), $\mathcal{M}(\xi)=$

$$
\left[\begin{array}{cccc}
-\sum_{j=1}^{N} \xi_{j} Q_{j} & \mathbf{M}_{\mathbf{3}}(\xi) & \mathbf{M}_{\mathbf{4}}(\xi) & \mathbf{M}_{\mathbf{7}}(\xi) \\
* & \mathbf{M}_{\mathbf{5}}(\xi) & \mathbf{M}_{\mathbf{6}}(\xi) & \sum_{i=1}^{N} \xi_{i} Z_{1 i}^{\prime} \\
* & * & -2 S_{\Delta} & \sum_{i=1}^{N} \xi_{i} Z_{2 i}^{\prime} \\
* & * & * & -2 \sum_{i=1}^{N} \xi_{i} S_{i}
\end{array}\right]<0
$$

em que o índice $j$ está associado ao instante $k+1$, os índices $i$ e $q$ estão associados ao instante $k$, e:

$$
\begin{gathered}
\mathbf{M}_{\mathbf{3}}(\xi)=\sum_{i=1}^{N} \xi_{i} A_{i} U+\sum_{i=1}^{N} \xi_{i} B_{i} \sum_{q=1}^{N} \xi_{q} Y_{1 q} \\
\mathbf{M}_{\mathbf{4}}(\xi)=\sum_{i=1}^{N} \xi_{i} G_{i} S_{\Delta}+\sum_{i=1}^{N} \xi_{i} B_{i} \sum_{q=1}^{N} \xi_{q} Y_{2 q} \\
\mathbf{M}_{\mathbf{5}}(\xi)=\sum_{i=1}^{N} \xi_{i} Q_{i}-U-U^{\prime} \\
\mathbf{M}_{\mathbf{6}}(\xi)=U^{\prime} \sum_{i=1}^{N} \xi_{i} L_{i}^{\prime} \Omega \quad e \\
\mathbf{M}_{\mathbf{7}}(\xi)=-\sum_{i=1}^{N} \xi_{i} B_{i} \sum_{q=1}^{N} \xi_{q} S_{q} .
\end{gathered}
$$

Considere, em particular o produto (no instante $k$ ):

$$
\begin{gathered}
B\left(\xi_{k}\right) Y_{1}\left(\xi_{k}\right)=\sum_{j=1}^{N} \xi_{j} \sum_{i=1}^{N} \xi_{i} B_{i} \sum_{q=1}^{N} \xi_{q} Y_{1 q}= \\
\sum_{j=1}^{N} \sum_{i=1}^{N} \xi_{j} \xi_{i}^{2} B_{i} Y_{1 i}+\sum_{j=1}^{N} \sum_{i=1}^{N-1} \sum_{q=i+1}^{N} \xi_{j} \xi_{i} \xi_{q}\left(B_{i} Y_{1 q}+B_{q} Y_{1 i}\right)
\end{gathered}
$$

Procedendo de forma similar para todos os termos envolvidos, pode-se escrever $\mathcal{M}(\xi)$ sob a forma equivalente

$\mathcal{M}(\xi)=\sum_{j=1}^{N} \sum_{i=1}^{N} \xi_{j} \xi_{i}^{2} \mathcal{M}_{j i}^{+}+\sum_{j=1}^{N} \sum_{i=1}^{N-1} \sum_{q=i+1}^{N} \xi_{j} \xi_{i} \xi_{q} \mathcal{M}_{j i q}^{+}<0$

na qual as matrizes $\mathcal{M}_{j i}^{+}$e $\mathcal{M}_{j i q}^{+}$são dadas em (19) e (20).

A partir de (19) e (20) tem-se, respectivamente, $\mathcal{M}_{j i}^{+}<0$ e $\mathcal{M}_{j i q}^{+}<0$, o que garante que (26) é verificada. Em consequência, a desigualdade (25) também é satisfeita. Como por hipótese a não-linearidade $\varphi\left(z_{k}\right)$ é válida globalmente e, por construção, $\Psi\left(u_{k}\right)$ é verificada $\forall x_{k} \in \mathcal{E}\left(\left(Q^{-1}(\xi)\right)\right)$, temos $\Delta V\left(x_{k}, \xi_{k}\right)=V\left(x_{k+1}, \xi_{k+1}\right)-\lambda V\left(x_{k}, \xi_{k}\right)<0 \mathrm{e}$, 
pela Definição 1, o CL $\mathcal{E}\left(\left(Q^{-1}(\xi)\right)\right)$ é robustamente absolutamente $\lambda$-contrativo. Consequentemente, para todo $x_{0} \in$ $S_{0} \triangleq \mathcal{E}\left(\left(Q^{-1}(\xi)\right)\right)=\bigcap_{i} \mathcal{E}\left\{Q_{i}^{-1}\right\}$, as trajetórias correspondentes do sistema (10) convergem assintoticamente para a origem para toda e qualquer não-linearidade $\varphi\left(z_{k}\right)$ verificando (3).

Observação 2 Considere a lei de controle independente de parâmetros utilizada em (Castelan et al., 2007) para tratar o caso em que $\xi_{k}$ não é mensurável em tempo real:

$$
u_{k}=K x_{k}+\Gamma \varphi\left(z_{k}\right) .
$$

Neste caso, os ganhos $K=Y_{1} U^{-1}$ e $\Gamma=Y_{2} S_{\Delta}^{-1}$ e o conjunto $\mathcal{S}_{0} \triangleq \mathcal{E}\left(Q^{-1}(\xi)\right)$ que solucionam o problema de estabilização (Problema 1) com controle a ganhos fixos correspondente, podem ser determinados se as desigualdades seguintes são factíveis:

$$
\begin{gathered}
{\left[\begin{array}{cccc}
-Q_{j} & A_{i} U+B_{i} Y_{1} & G_{i} S_{\Delta}+B_{i} Y_{2} & -B_{i} S_{i} \\
\star & \lambda \mathbf{M}_{3 \mathbf{i}} & U^{\prime} L_{i}^{\prime} \Omega & Z_{1 i}^{\prime} \\
\star & \star & -2 S_{\Delta} & Z_{2 i}^{\prime} \\
\star & \star & \star & -2 S_{i}
\end{array}\right]<0} \\
\forall i=1, \ldots, N e \forall j=1, \ldots, N \\
{\left[\begin{array}{ccc}
-Q_{i}+U+U^{\prime} & -U^{\prime} L_{i}^{\prime} \Omega & Y_{1(\ell)}^{\prime}-Z_{1 i(\ell)}^{\prime} \\
\star & 2 S_{\Delta} & Y_{2(\ell)}^{\prime}-Z_{2 i(\ell)}^{\prime} \\
\star & \star & \rho_{(\ell)}^{2}
\end{array}\right] \geq 0} \\
\forall i=1, \ldots, N e \forall \ell=1, \ldots, m
\end{gathered}
$$

Quando $\lambda=1$, o resultado acima corresponde ao proposto em (Castelan et al., 2007).

O caso em que as matrizes do sistema não são incertas pode ser tratado utilizando-se o mesmo arcabouço, mas considerando-se a contratividade associada à um só conjunto elipsoidal $\mathcal{E}\left(Q^{-1}\right)$ (Castelan et al., 2006).

\subsection{Síntese via programação convexa}

Para a otimização do tamanho do conjunto $\mathcal{S}_{0} \triangleq \mathcal{E}\left(Q^{-1}(\xi)\right)$, nós adotamos, como em (Castelan et al., 2006; Gomes da Silva Jr. et al., 2004; Hu et al., 2002; Ghaoui and Scorletti, 1996) um conjunto modelo para síntese. Assim, considere um conjunto modelo poliedral dado por $\mathcal{X}_{0} \subset \Re^{n}$ e o fator escalar $\beta \in \Re: \mathcal{X}_{0} \triangleq \operatorname{Co}\left\{v_{r} \in \Re^{n}, r=1, \ldots, n_{r}\right\}$. A meta consiste em maximizar o fator escalar $\beta$ tal que a seguinte condição de inclusão seja verificada:

$$
\begin{aligned}
\beta \mathcal{X}_{0} \subset \mathcal{E}\left(Q^{-1}(\xi)\right) & \Longleftrightarrow\left(\beta v_{r}^{\prime}\right) Q^{-1}(\xi)\left(\beta v_{r}\right) \leq 1 \\
\forall r & =1, \ldots, n_{r}, \forall \xi \in \Xi
\end{aligned}
$$

Através do complemento de Schur, a verificação de (30) é equivalente à seguinte restrição convexa:

$$
\left[\begin{array}{cc}
\mu & v_{r}^{\prime} \\
v_{r} & Q_{i}
\end{array}\right] \geq 0 \quad \begin{aligned}
& \forall i=1, \ldots, N \\
& \forall r=2, \ldots, n_{r}
\end{aligned}
$$

na qual, por definição, $\mu=\frac{1}{\beta^{2}}$. Então, podemos formular o problema de programação convexa seguinte para a síntese do controlador dependente de parâmetros:

$$
\begin{gathered}
\min _{Q_{i}, U, S_{\Delta}, S_{i}, Z_{1 i}, Z_{2 i}, Y_{1 i}, Y_{2 i},}{ }^{\text {sujeito a }} \\
\text { LMIs (19), (20), (21) e (31) }
\end{gathered}
$$

Com base na Observação 2, um problema de programação convexa similar ao problema (32) pode ser utilizado para a síntese da lei de controle a ganhos fixos:

$$
\begin{gathered}
\min _{Q_{i}, U, S_{\Delta}, S_{i}, Z_{1 i}, Z_{2 i}, Y_{1}, Y_{2},}{ }^{\text {sujeito a }} \\
\text { LMIs (28), (29) e (31) }
\end{gathered}
$$

Note que a região $\beta \mathcal{X}_{0}$ obtida a partir de (32) ou (33) é uma região de estabilidade assintótica associada ao sistema em malha fechada pois, por construção, $\beta \mathcal{X}_{0}$ encontra-se no interior do domínio de contratividade $\mathcal{E}\left(Q^{-1}(\xi)\right)$. Isto garante que $\lim _{k \rightarrow \infty} x_{k}=0, \forall x_{0} \in \beta \mathcal{X}_{0}$, muito embora parte das trajetórias que emanam desta região possam evoluir no seu exterior.

Além disso, outra possibilidade é escolher o conjunto modelo sob a forma elipsoidal, $\mathcal{X}_{0} \triangleq \mathcal{E}\left(R^{-1}\right)$, em que $R=R^{\prime}>0$ é escolhida à priori, da mesma forma que os valores de $v_{r}$ do conjunto modelo poliedral (Hu et al., 2002). No caso elipsoidal pode-se substituir a restrição convexa (31) por: $\left[\begin{array}{cc}\mu R & I \\ I & Q_{i}\end{array}\right] \geq 0, \forall i=1, \ldots, N$.

Note que restrições convexas adicionais podem ser consideradas nos problemas de otimização anteriores como, por exemplo, para tratar o caso em que a condição de setor (3) é localmente verificada.

Observação 3 Se a condição (3) é válida localmente, ou seja, somente em $\mathcal{Z} \subset \Re^{p}$ definido por

$$
\begin{aligned}
\mathcal{Z} & =\left\{z_{k} \in \Re^{p} ;\left|z_{k}\right| \leq \nu\right\}, \operatorname{com} \nu_{(q)}>0, q=1, \ldots, p \\
& =\left\{x_{k} \in \Re^{n} ;\left|L\left(\xi_{k}\right) x_{k}\right| \leq \nu, \forall \xi_{k} \in \Xi\right\} .
\end{aligned}
$$

Então, considera-se a condição adicional $\mathcal{E}\left(Q^{-1}\left(\xi_{k}\right)\right) \subset \mathcal{Z}$, dada por

$$
\left[\begin{array}{cc}
Q_{i} & L_{i(q)}^{\prime} \\
* & \nu_{(q)}^{2}
\end{array}\right] \geq 0 \forall q=1, \ldots, p
$$


Esta condição adicional pode restringir o tamanho do conjunto $\mathcal{S}_{0}$, comparativamente ao caso em que $\varphi\left(z_{k}\right)$ é globalmente verificada, como relatado na seção seguinte.

Finalmente, é importante observar que as restrições de inclusão (21) e (29) permitem explorar efetivamente a saturação da ação de controle para o atendimento dos requisitos de projeto. Uma técnica similar é proposta em (Ghaoui and Scorletti, 1996) e utilizada em (Ghaoui and Niculescu, 2000) para o caso de sistemas contínuos no tempo. Entretanto, esta técnica utiliza restrições de inclusão que, diferentemente de (21) e (29), não consideram que as entradas de controle possam saturar. Note também que a abordagem proposta no presente trabalho permite considerar, conjuntamente ou de forma isolada, não-linearidades que podem ser representadas sob a forma de parâmetros variantes e/ou que podem ser consideradas pertencentes a um setor. Deste ponto de vista, nossa abordagem tem um caráter mais abrangente do que a técnica utilizada em (Ghaoui and Scorletti, 1996) e (Ghaoui and Niculescu, 2000).

\section{EXEMPLO NUMÉRICO}

Para ilustrar a aplicabilidade dos resultados teóricos e do algoritmo da síntese propostos, considere os dados seguintes para o sistema (1)-(3) (Castelan et al., 2007):

$$
\begin{aligned}
& A_{1}=\left[\begin{array}{ll}
-1.1 & 0.4 \\
-0.2 & 1.1
\end{array}\right], A_{2}=\left[\begin{array}{rr}
-0.2 & 0.7 \\
0.6 & 1.3
\end{array}\right] \text {, } \\
& B_{1}=\left[\begin{array}{r}
0 \\
1.2
\end{array}\right], B_{2}=\left[\begin{array}{r}
0 \\
1.3
\end{array}\right], G_{1}=G_{2}=\left[\begin{array}{l}
1 \\
0
\end{array}\right], \\
& L_{1}=\left[\begin{array}{ll}
0 & 1.1
\end{array}\right], L_{2}=\left[\begin{array}{ll}
0 & 0.9
\end{array}\right] \text {, } \\
& \Omega=0.7, \rho=1 \text { e } \varphi=0.3 z(1+\operatorname{sen}(z)), \forall z \in \Re^{p}
\end{aligned}
$$

Considere também:

$$
\mathcal{X}_{0}=\operatorname{Co}\left\{\left[\begin{array}{l}
1 \\
0
\end{array}\right],\left[\begin{array}{l}
0 \\
1
\end{array}\right],\left[\begin{array}{r}
-1 \\
0
\end{array}\right],\left[\begin{array}{r}
0 \\
-1
\end{array}\right]\right\} .
$$

Os resultados numéricos apresentados na Tabela 1 , referemse à lei de controle dependente de parâmetros (6) e foram obtidos utilizando (32). Na Figura 1, temos as representações dos conjuntos $\mathcal{E}\left(Q_{1}^{-1}\right)$ e $\mathcal{E}\left(Q_{2}^{-1}\right)$. Na Figura 1(a), a interseção das elipses externas, em linhas cheias, corresponde à solução $\mathcal{S}_{0}$ para $\lambda=0.95$, e a interseção das elipses internas, em linhas tracejadas, para $\lambda=0.8 \mathrm{e}$, da mesma forma, para $\lambda=0.95$ e $\lambda=0.6405$ na Figura 1(b). Pode-se observar que com a exigência de melhor desempenho (ou seja, menor co-

\begin{tabular}{|c|c|c|}
\hline & $\lambda=0.95$ & $\lambda=0.6405$ \\
\hline \multirow{2}{*}{$Q_{1}$} & $\begin{array}{ll}3.9365 & 1.7751\end{array}$ & $0.6468 \quad 0.5442$ \\
\hline & $1.7751 \quad 2.0421$ & $0.5442 \quad 0.5864$ \\
\hline \multirow{2}{*}{$Q_{2}$} & $\begin{array}{ll}3.0956 & 0.7287\end{array}$ & $\begin{array}{ll}0.6882 & 0.3184\end{array}$ \\
\hline & $0.7287 \quad 1.4142$ & $0.3184 \quad 0.2757$ \\
\hline$K_{1}$ & $-0.3065-0.7634$ & $-0.6062-0.6361$ \\
\hline$K_{2}$ & $-0.3892-0.9111$ & $-0.4766-0.7237$ \\
\hline$\overline{\Gamma_{1}}$ & -0.0021 & 0.8488 \\
\hline$\overline{\Gamma_{2}}$ & 0.2459 & 0.2079 \\
\hline$\beta$ & 1.1140 & 0.3583 \\
\hline
\end{tabular}
eficiente de contratividade) ocorre uma diminuição da região em que a estabilidade assintótica é garantida. $\mathrm{O}$ menor valor de $\lambda$ para o qual o problema (32) é factível é $\lambda=0.6405$.
Tabela 1: Resultados numéricos - $u_{k}=K(\xi) x_{k}+\Gamma(\xi) \varphi$

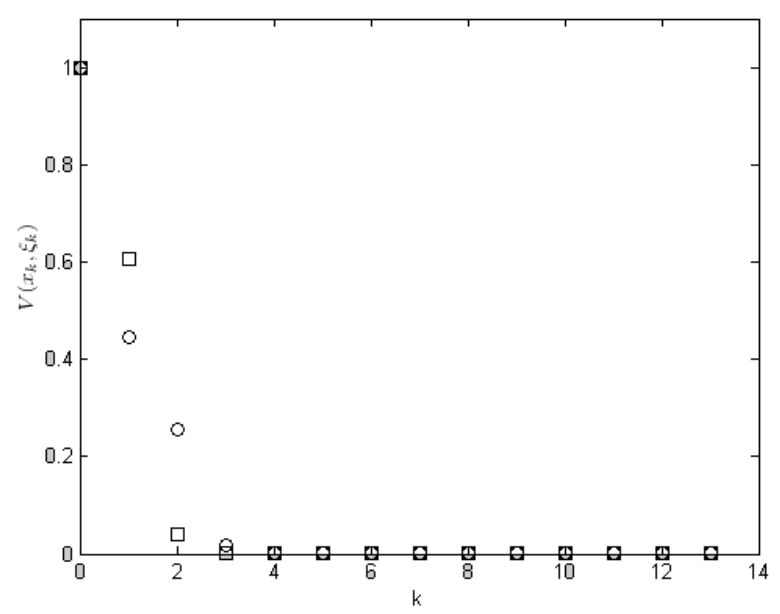

Figura 3: Valores de $V\left(x_{k}, \xi_{k}\right)$ ao longo das trajetórias dos estados da Figura 2 (a) e (b), para as duas sequências $\left\{\xi_{k}\right\}_{1}(\square)$ e $\left\{\xi_{k}\right\}_{2}(\circ)$

A simulação do sistema sob saturação, para $\lambda=0.95$ e a condição inicial $x_{0}=[0.63881 .1891]^{\prime}$ assinalada na Figura 1(a), é mostrada na Figura 2 utilizando-se duas sequências diferentes de parâmetros, as quais são denotadas $\left\{\xi_{k}\right\}_{1}$ e $\left\{\xi_{k}\right\}_{2}$ e apresentadas no Anexo I. Nos gráficos à direita, representamos o valor de controle calculado, $u_{k}$ (representado por $*)$, e o valor de controle efetivamente aplicado, $\operatorname{sat}\left(u_{k}\right)$ (representado por $\square$ ). Cabe salientar que a saturação da ação de controle observada para estas duas sequências de parâmetros pode não ocorrer para outras sequências particulares. Nos gráficos à esquerda observa-se a convergência assintótica para a origem das trajetórias dos estados. A Figura 3 mostra o desempenho do sistema em termos dos valores de $V\left(x_{k}, \xi_{k}\right)$, calculado ao longo destas trajetórias.

$\mathrm{Na}$ Figura 4, a interseção das elipses em linhas pontilhadas representam os conjuntos $\mathcal{S}_{0}$ obtidos, via o problema de otimização (33), para as leis de controle a parâmetros fixos (27) 

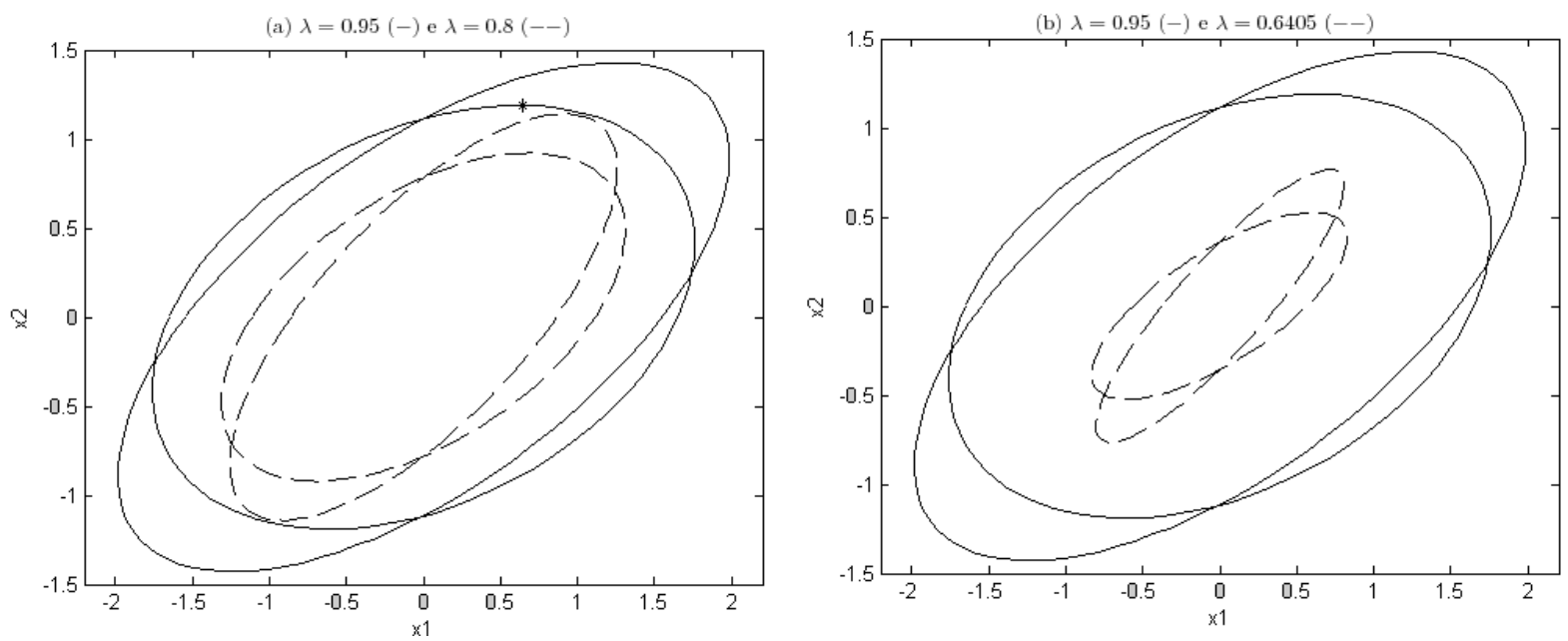

Figura 1: Conjuntos $\mathcal{E}\left(Q_{1}^{-1}\right)$ e $\mathcal{E}\left(Q_{2}^{-1}\right)$ para $u_{k}=K(\xi) x_{k}+\Gamma(\xi) \varphi\left(z_{k}\right)$
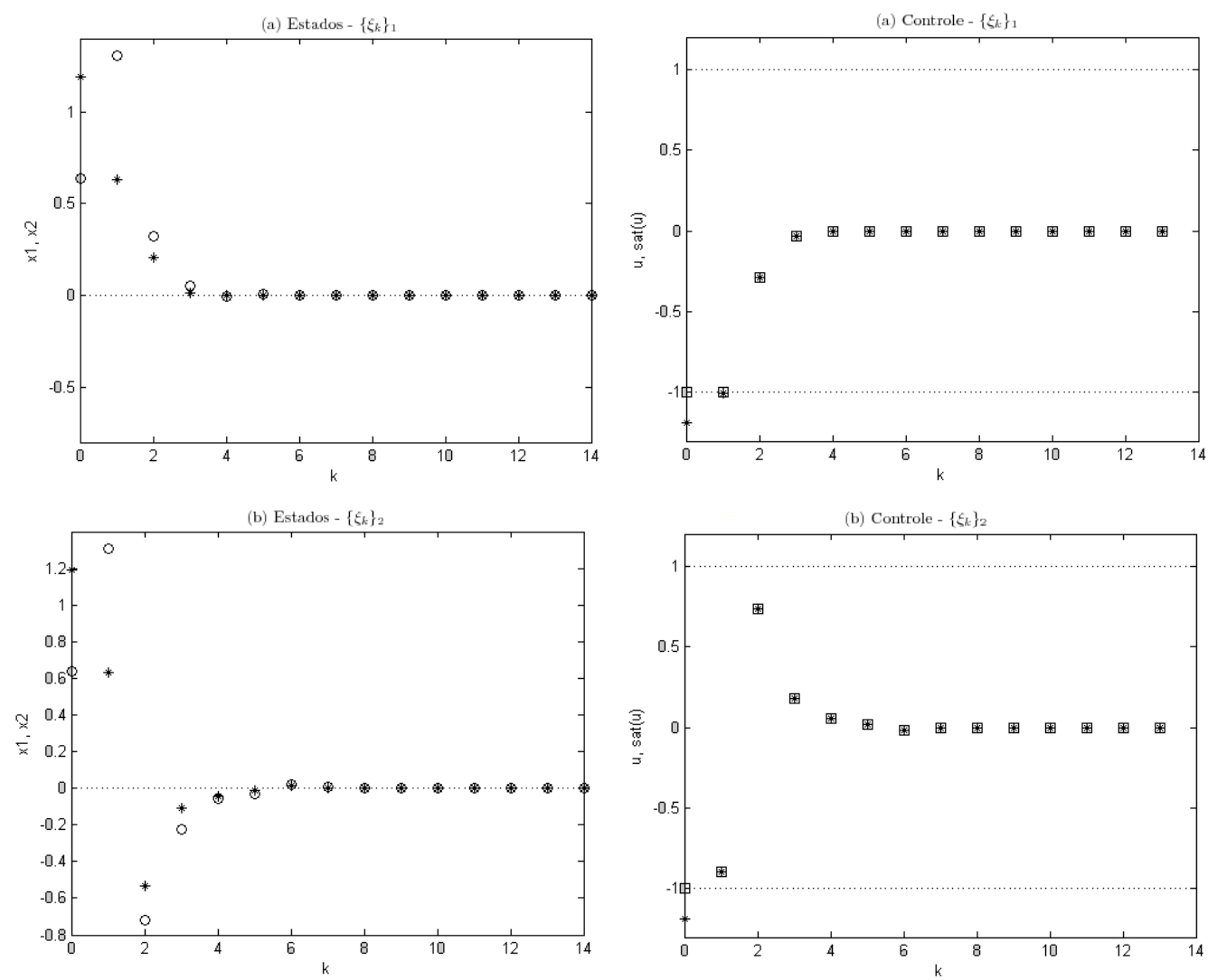

Figura 2: Simulações para duas sequências aleatórias diferentes, $\left\{\xi_{k}\right\}_{1}$ e $\left\{\xi_{k}\right\}_{2}$, com $x_{0}=[0.63881 .1891]^{\prime}, \lambda=0.95$ e $u_{k}=K(\xi) x_{k}+\Gamma(\xi) \varphi\left(z_{k}\right)$ 

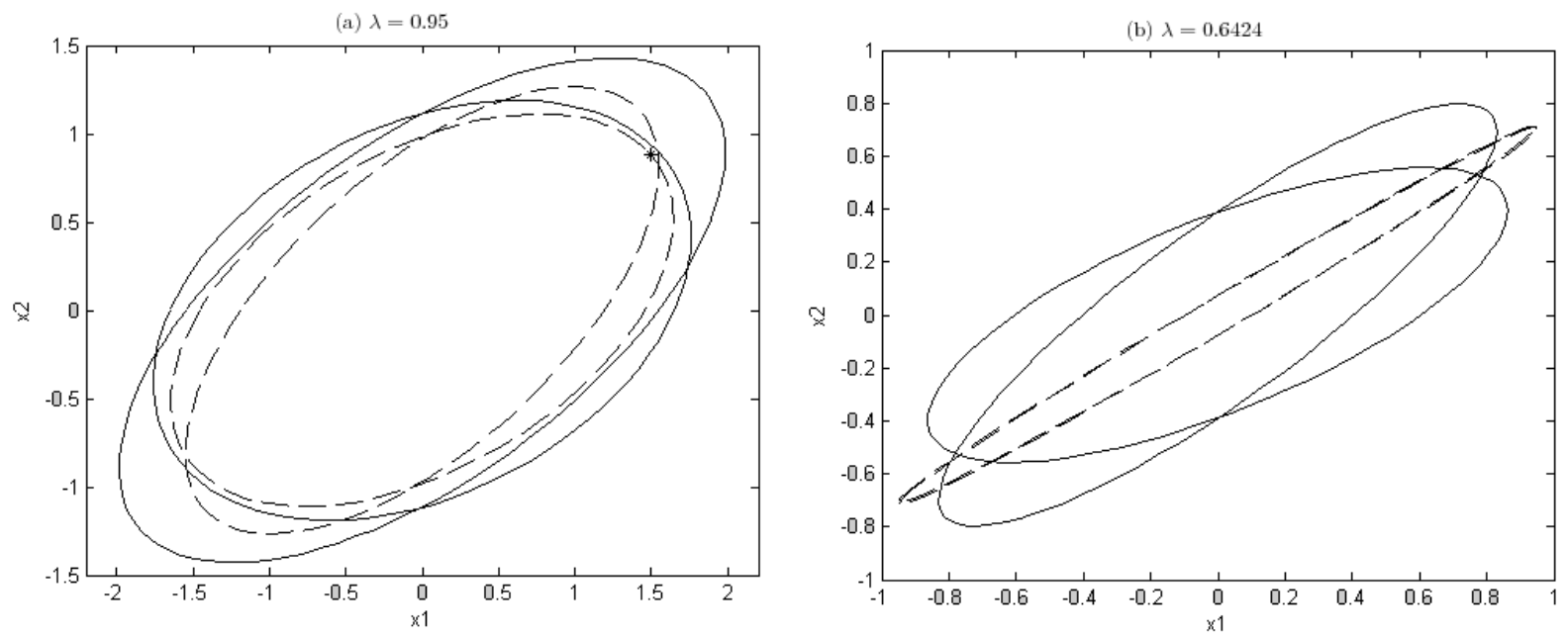

Figura 4: Conjuntos $\mathcal{E}\left(Q_{1}^{-1}\right)$ e $\mathcal{E}\left(Q_{2}^{-1}\right)$ para $u_{k}=K(\xi) x_{k}+\Gamma(\xi) \varphi\left(z_{k}\right)(-)$ e $u_{k}=K x_{k}+\Gamma \varphi\left(z_{k}\right)(--)$
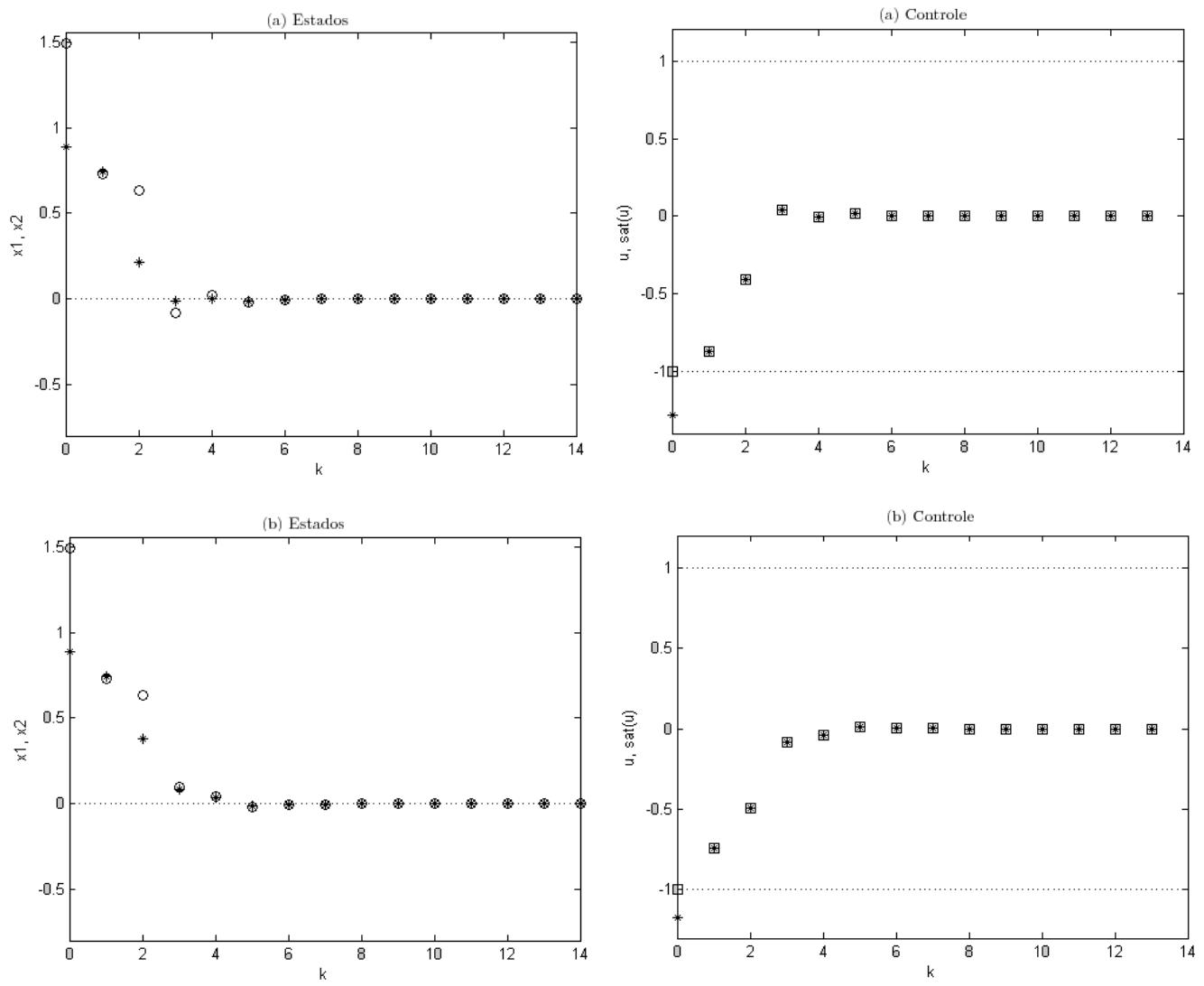

Figura 5: Simulações com $\lambda=0.95,\left\{\xi_{k}\right\}_{1}$ e $x_{0}=\left[\begin{array}{lll}1.4911 & 0.8850\end{array}\right]^{\prime}$ para: (a) $u_{k}=K(\xi) x_{k}+\Gamma(\xi) \varphi\left(z_{k}\right)$ e (b) $u_{k}=K x_{k}+\Gamma \varphi\left(z_{k}\right)$ 
Tabela 2: Resultados numéricos - $u_{k}=K x_{k}+\Gamma \varphi\left(z_{k}\right)$

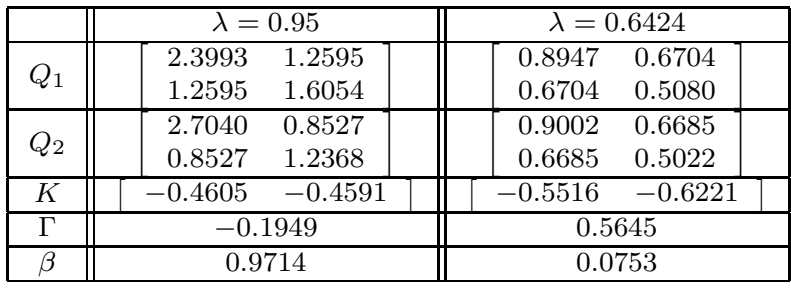

calculadas para $\lambda=0.95$ e $\lambda=0.6424$, conforme Tabela 5. O menor valor factível para $\lambda$-contratividade, com a lei de controle a parâmetros fixos, é $\lambda=0.6424$. A interseção das elipses em linhas cheias representam os conjuntos $\mathcal{S}_{0}$ obtidos via o problema de otimização (32), associados às leis de controle dependentes de parâmetros calculadas para os mesmos coeficientes de contratividade. Observamos que a lei de controle dependente de parâmetros permite obter maiores regiões de estabilidade para o sistema em malha fechada, comparativamente à lei de controle a parâmetros fixos. Além disso, esta diferença aumenta com a diminuição do coeficiente de contratividade.

Na Figura 5 mostram-se os resultados de simulações para o sistema em malha fechada sob a ação da lei de controle a parâmetros variantes e fixos, considerando $x_{0}=$ $\left[\begin{array}{ll}1.4911 & 0.8850\end{array}\right]^{\prime}$ indicada na Figura 4(a), $\lambda=0.95$ e a sequência $\left\{\xi_{k}\right\}_{1}$. Na parte superior, Figura 5(a), consideramos os ganhos a parâmetros variantes e, na parte inferior, Figura 5(b), temos o resultado da simulação quando os ganhos são a parâmetros fixos. É importante salientar que a utilização de outras sequências de parâmetros e/ou de outras condições iniciais implicarão em desempenhos distintos para cada caso. Na Figura 6, encontramos o comparativo do desempenho do sistema em termos dos valores de $V\left(x_{k}, \xi_{k}\right)$ para a lei de controle a parâmetros variantes e fixos, ao longo destas trajetórias.

Na Figura 7 são mostrados os conjuntos elipsoidais obtidos ao calcular-se $u_{k}=K(\xi) x_{k}+\Gamma(\xi) \varphi\left(z_{k}\right)$ e $u_{k}=K(\xi) x_{k}$, para $\lambda=0.8$ e $\lambda=0.6627$, via o problema de otimização (32). Considerou-se $Y_{2 i}, Z_{2 i}=0$ para o caso em que deseja-se $\Gamma(\xi)=0$ e o menor valor factível obtido para $\lambda$-contratividade neste caso foi $\lambda=0.6627$, conforme Tabela 5. A diminuição do conjunto $\mathcal{S}_{0}$ associado a $u_{k}=K(\xi) x_{k}$ fica mais evidenciada para valores menores de $\lambda$. Na Figura 8 apresenta-se a simulação do sistema em malha fechada usando o controle sem a realimentação da não-linearidade calculada para $\lambda=0.95$, com a condição inicial $x_{0}=[0.63881 .1891]^{\prime}$, indicada na Figura 1(a), e a sequência $\left\{\xi_{k}\right\}_{1}$; neste caso, o conjunto $\mathcal{S}_{0}$ obtido tende ao conjunto $\mathcal{S}_{0}$ associado à lei de controle com realimentação da não-linearidade, correspondente à interseção das elipses

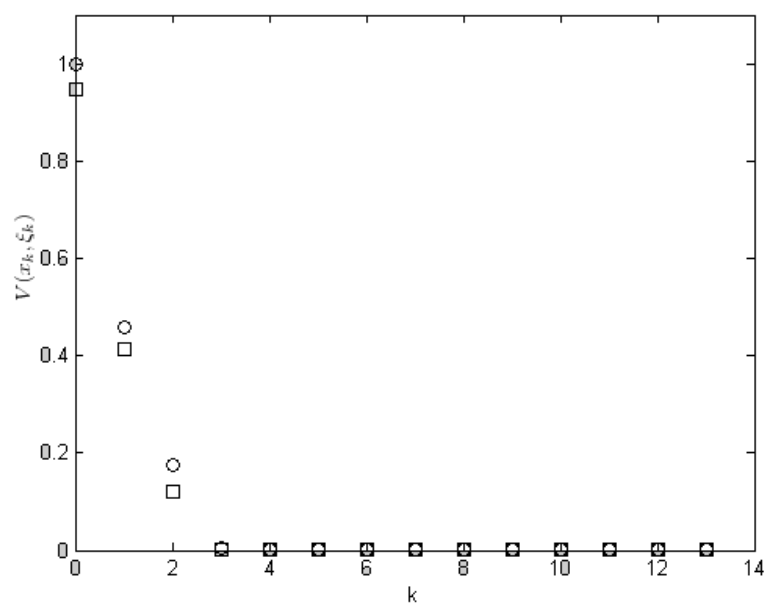

Figura 6: Valores de $V\left(x_{k}, \xi_{k}\right)$ ao longo das trajetórias dos estados para $u_{k}=K(\xi) x_{k}+\Gamma(\xi) \varphi\left(z_{k}\right)-(\square)$ e $u_{k}=K x_{k}+$ $\Gamma \varphi\left(z_{k}\right)-(\circ), \operatorname{com} \lambda=0.95,\left\{\xi_{k}\right\}_{1}$ e $x_{0}=\left[\begin{array}{lll}1.4911 & 0.8850\end{array}\right]^{\prime}$

Tabela 3: Resultados numéricos - $u_{k}=K(\xi) x_{k}$

\begin{tabular}{|c|c|c|c|c|}
\hline & \multicolumn{2}{|c|}{$\lambda=0.95$} & \multicolumn{2}{|c|}{$\lambda=0.6627$} \\
\hline \multirow[b]{2}{*}{$Q_{1}$} & 3.6669 & 1.5859 & 0.4792 & 0.3796 \\
\hline & 1.5859 & 1.8462 & 0.3796 & 0.4459 \\
\hline \multirow{2}{*}{$Q_{2}$} & 3.1211 & 0.6133 & 0.5263 & 0.2729 \\
\hline & 0.6133 & 1.2825 & 0.2729 & 0.2287 \\
\hline$\overline{K_{1}}$ & -0.2578 & -0.8953 & -0.9090 & -0.1019 \\
\hline$K_{2}$ & -0.4062 & -0.7433 & -0.5545 & -0.4522 \\
\hline$\beta$ & \multicolumn{2}{|c|}{1.0768} & \multicolumn{2}{|c|}{0.2952} \\
\hline
\end{tabular}

em linha cheia da Figura 1(a). Verifica-se que o desempenho temporal é similar utilizando-se as duas leis de controle com e sem a realimentação da não-linearidade (Figuras 2(a) e 8 , respectivamente); isto também pode ser observado na Figura 9(a) que mostra o desempenho do sistema em termos dos valores de $V\left(x_{k}, \xi_{k}\right)$. Através de simulações, verificamos que a realimentação da não-linearidade pode proporcionar melhor desempenho à medida que calculam-se as leis de controle para menores coeficientes de $\lambda$-contratividade. A Figura 9(b) mostra o desempenho em termos de $V\left(x_{k}, \xi_{k}\right)$ ao longo das trajetórias obtidas com as leis de controle calculadas para $\lambda=0.6627$, com a sequência $\left\{\xi_{k}\right\}_{1}$, e $x_{0}=$ [0.5778 0.4782], conforme assinalada na Figura 7(b).

Finalmente, com o objetivo de ilustrar a Observação 3, a Figura 10 mostra, em linhas pontilhadas, os conjuntos elipsoidais obtidos ao calcular-se $u_{k}=K(\xi) x_{k}+\Gamma(\xi) \varphi\left(z_{k}\right)$ para $\varphi\left(z_{k}\right)$ localmente verificada, $z_{k} \in \mathcal{Z} \subset \Re^{p}, \operatorname{com} \nu=10$. Neste caso, o menor valor factível para o coeficiente de contratividade é $\lambda=0.731$. Em linhas cheias são mostrados os conjuntos elipsoidais obtidos quando $\varphi\left(z_{k}\right)$ é globalmente verificada, $\mathcal{Z}=\Re^{p}$. Consequentemente, a região de estabili- 

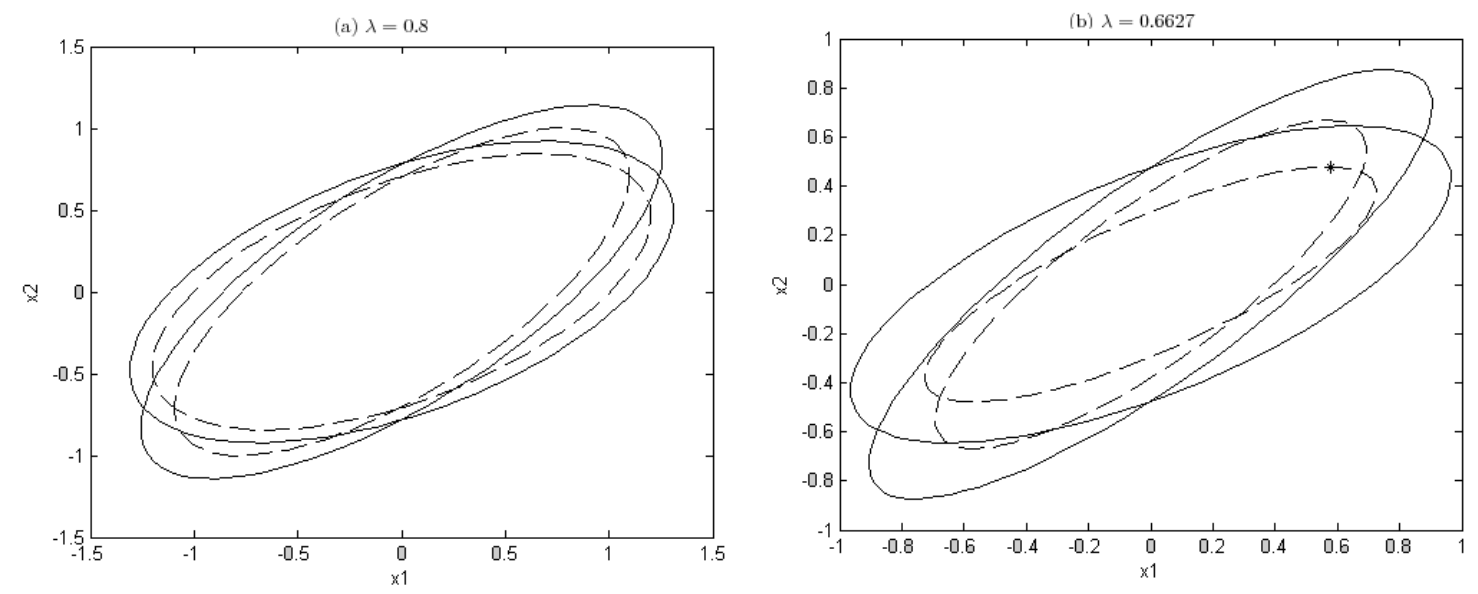

Figura 7: Conjuntos $\mathcal{E}\left(Q_{1}^{-1}\right)$ e $\mathcal{E}\left(Q_{2}^{-1}\right)$ para $u_{k}=K(\xi) x_{k}+\Gamma(\xi) \varphi\left(z_{k}\right)(-)$ e $u_{k}=K(\xi) x_{k}(--)$
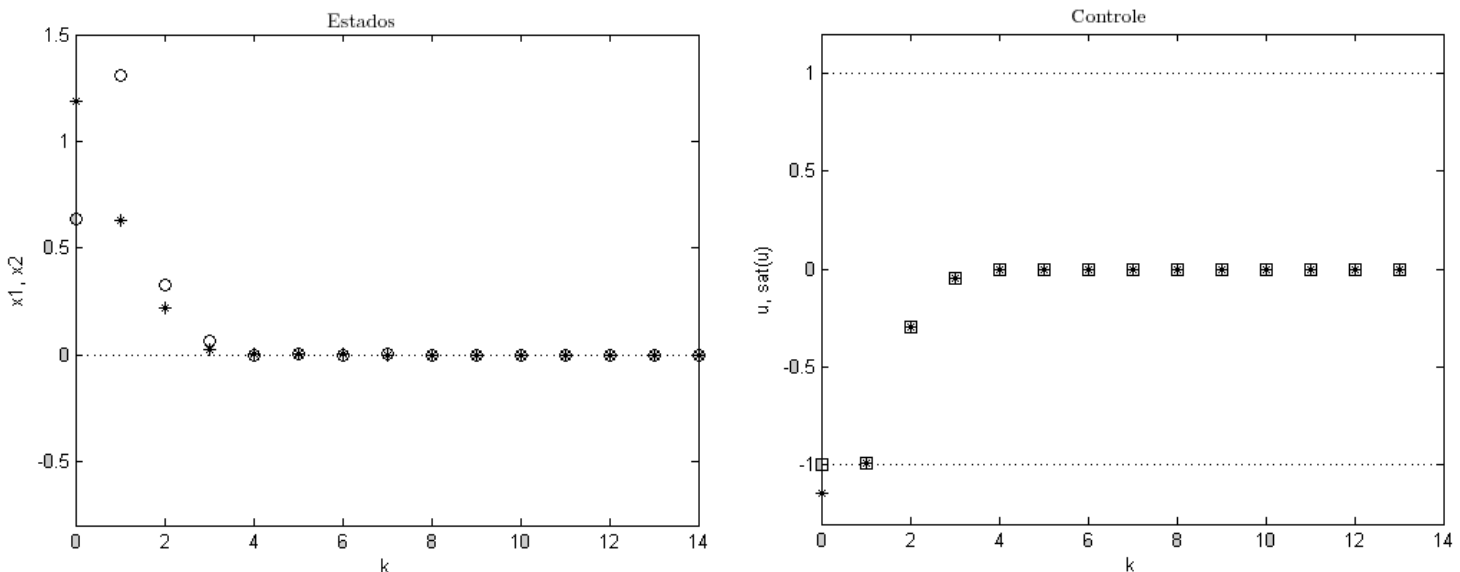

Figura 8: Simulação para $u_{k}=K(\xi) x_{k}$, calculada para $\lambda=0.95$, com $\left\{\xi_{k}\right\}_{1}$ e $x_{0}=[0.63881 .1891]^{\prime}$
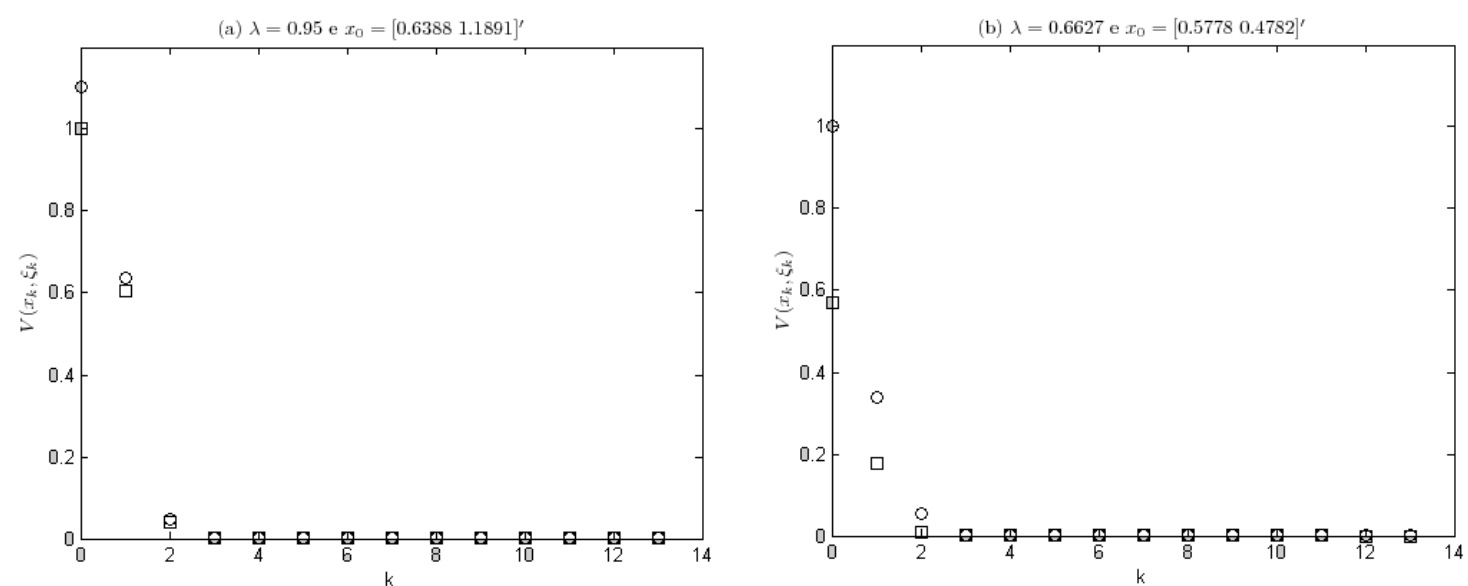

Figura 9: Valores de $V\left(x_{k}, \xi_{k}\right)$ ao longo das trajetórias dos estados para $u_{k}=K(\xi) x_{k}+\Gamma(\xi) \varphi\left(z_{k}\right)$ - (口) e $u_{k}=K(\xi) x_{k}$ - (०) $\operatorname{com}\left\{\xi_{k}\right\}_{1}$ 

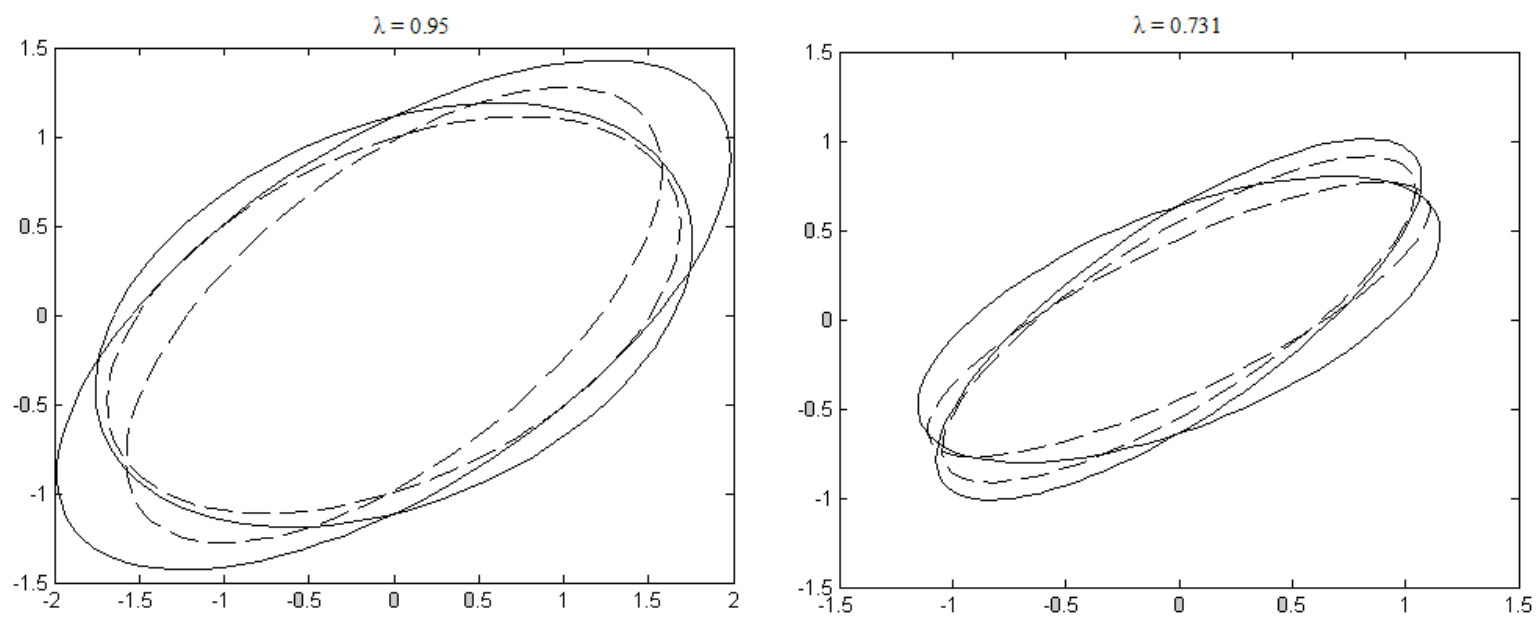

Figura 10: Conjuntos $\mathcal{E}\left(Q_{1}^{-1}\right)$ e $\mathcal{E}\left(Q_{2}^{-1}\right)$ para $u_{k}=K(\xi) x_{k}+\Gamma(\xi) \varphi\left(z_{k}\right)$ para $\varphi(\cdot)$ localmente verificada

Tabela 4: Resultados numéricos para $\varphi(\cdot)$ localmente verificada

\begin{tabular}{|c|c|c|c|c|}
\hline & \multicolumn{2}{|c|}{$\lambda=0.95$} & \multicolumn{2}{|c|}{$\lambda=0.731$} \\
\hline \multirow{2}{*}{$Q_{1}$} & 2.4815 & 1.2812 & 1.1481 & 0.7879 \\
\hline & 1.2812 & 1.6305 & 0.7879 & 0.8280 \\
\hline \multirow{2}{*}{$Q_{2}$} & 2.8388 & 0.8371 & 1.3260 & 0.6825 \\
\hline & 0.8371 & 1.2368 & 0.6825 & 0.6391 \\
\hline$K_{1}$ & -0.2537 & -0.7851 & -0.4165 & -0.7331 \\
\hline$\overline{K_{2}}$ & -0.5599 & -0.7653 & -0.5927 & -0.6225 \\
\hline$\Gamma_{1}$ & \multicolumn{2}{|c|}{-3.8447} & \multicolumn{2}{|c|}{-10.1242} \\
\hline$\Gamma_{2}$ & \multicolumn{2}{|c|}{-2.5841} & \multicolumn{2}{|c|}{-10.0296} \\
\hline$\beta$ & \multicolumn{2}{|c|}{0.9842} & \multicolumn{2}{|c|}{0.5357} \\
\hline
\end{tabular}

dade garantida $\mathcal{S}_{0} \subset \mathcal{E}\left(Q_{i}^{-1}\right)$ é mais restrita quando $\varphi\left(z_{k}\right)$ é localmente verificada. Os dados numéricos correspondentes encontram-se na Tabela 5.

\section{CONCLUSÕES}

Neste trabalho aplicou-se o conceito de $\lambda$-contratividade para tratar o problema de síntese de uma lei de controle dependente de parâmetros para uma classe de sistemas nãolineares sob atuadores saturantes. A abordagem utilizada permite considerar não-linearidades que podem ser representadas como parâmetros variantes e/ou não-linearidades de tipo setor globalmente ou localmente verificadas.

O exemplo numérico considerado teve por objetivo mostrar, em particular, que o uso de ganhos variantes e a realimentação da não-linearidade permitem encontrar resultados menos conservadores do que nos casos sem realimentação da nãolinearidade de setor ou no caso a ganhos fixos, no que se refere ao tamanho da região $\mathcal{S}_{0}$ onde é garantida a estabilidade. Também procuramos evidenciar o compromisso exis- tente entre o tamanho de $\mathcal{S}_{0}$ e o desempenho associado a $\lambda$.

Trabalhos futuros apontam para: i) a solução do problema de controle via a utilização de compensadores dinâmicos com ganhos dependentes de parâmetros; ii) o aprimoramento dos algoritmos visando a obtenção de resultados menos conservadores no que se refere à região de estabilidade garantida, $\mathcal{S}_{0}$, particularmente considerando que o parâmetro incerto tem variação limitada e/ou utilizando técnicas menos conservadoras para a solução de LMIs dependente de parâmetros; e iii) a utilização e a adaptação desta abordagem para a solução de problemas práticos.

\section{AGRADECIMENTOS}

Este trabalho teve suporte financeiro parcial do CNPq. O primeiro autor é professor do Departamento de Matemática - CCT - UDESC/SC, bolsista PICDT - Capes. Os autores agradecem a Marc Jungers (CRAN/ENSEM, França) pelas discussões e aos revisores pelos comentários e sugestões.

\section{ANEXO I}

Sequências de parâmetros usadas no exemplo:

$\left\{\xi_{k}\right\}_{1}=\{0,0.0888,0.2825,0.1866,0.9635,0.0563$, $0.1753,0.4410,0.7579,0.9288,0.3496,0.2224,0.0524$, $0.6427,0.4539,0.4110,0.5076,0.6418,0.8435,0.5471\}$;

$\left\{\xi_{k}\right\}_{2}=\{0,0.9,0.1,0,0.0138,0.9875,0.1740$, $0.4030,0.3993,1,1,0.9,0.1,0,0.7857$, $0.5059,0.5624,0.7012\}$; 


\section{ANEXO II}

Demonstração do Lema 2:

$$
\begin{gathered}
x \in L_{V} \Leftrightarrow \forall \xi_{k} \in \Xi, \quad V\left(x_{k}, \xi_{k}\right)<1 \Leftrightarrow \\
x \in \bigcap_{\xi_{k} \in \Xi}\left\{\mathcal{E}\left(Q^{-1}\left(\xi_{k}\right)\right)\right\}
\end{gathered}
$$

Além disso, necessariamente, $\bigcap_{\xi_{k} \in \Xi}\left\{\mathcal{E}\left(Q^{-1}\left(\xi_{k}\right)\right)\right\} \subset$ $\bigcap_{i \in\{1, \ldots, N\}} \mathcal{E}\left(Q_{i}^{-1}\right)$. Por outro lado, para provar que $\bigcap_{i \in\{1, \ldots, N\}} \mathcal{E}\left(Q_{i}^{-1}\right) \subset \bigcap_{\xi_{k} \in \Xi}\left\{\mathcal{E}\left(Q^{-1}\left(\xi_{k}\right)\right)\right\}$, considere $x \in$ $\bigcap \mathcal{E}\left(Q_{i}^{-1}\right)$, então $\forall i=1, \ldots, N, x^{\prime} Q_{i}^{-1} x<$ $i \in\{1, \ldots, N\}$

1 , o qual, pelo complemento de Schur, é equivalente a $\left[\begin{array}{cc}1 & x^{\prime} \\ x & Q_{i}\end{array}\right]>0$. Assim $\forall \xi_{k} \in \Xi, \quad\left[\begin{array}{cc}1 & x^{\prime} \\ x & Q\left(\xi_{k}\right)\end{array}\right]>0$. Isto implica que $x \in \mathcal{E}\left(Q^{-1}\left(\xi_{k}\right)\right), \forall \xi_{k} \in \Xi$, ou $x \in$ $\bigcap_{\xi} \mathcal{E}\left(Q^{-1}\left(\xi_{k}\right)\right)$.

\section{REFERÊNCIAS}

Arcak, M. and Kokotovic, P. (2001). Feasibility conditions for circle criterion designs, Systems \& Control Letters 42(5): 405-412.

Arcak, M., Larsen, M. and Kokotovic, P. (2003). Circle and popov criteria as tools for nonlinear feedback designs, Automatica 39: 643-650.

Castelan, E. B., Corso, J. and Moreno, U. F. (2007). Stability and stabilization of a class of uncertain nonlinear discrete-time systems with saturating actuators, Symposium on System, Structure and Control, SSSC2007, Foz do Iguaçu - PR, Brazil.

Castelan, E. B., Moreno, U. and de Pieri, E. R. (2006). Absolute stabilization of discrete-time systems with a sector bounded nonlinearity under control saturations, IEEE International Symposium on Circuits and Systems (ISCAS 2006), Greece, pp. 3105-3108.

Castelan, E. B., Tarbouriech, S. and Queinnec, I. (2005). Stability and stabilization of a class of nonlinear systems with saturating actuators, Proc. of 16th IFAC World Congress in Automatic Control, Prague.

Castelan, E. B., Tarbouriech, S. and Queinnec, I. (2008). Control design for a class of nonlinear continuous-time systems, Automatica 44(8): 2034-2039.

Ghaoui, L. E. and Niculescu, S. L. (2000). Avances in linear matrix inequality methods in control, Siam.
Ghaoui, L. E. and Scorletti, G. (1996). Control of rational systems using linear-fractional representations and linear matrix inequalities, Automatica 32(9): 1273-1284.

Ghiggi, I. and Gomes da Silva Jr., J. M. (2006). Análise de estabilidade de sistemas lineares com atraso apresentando saturação de controle, XVI Brazilian Automatic Control Conference, Salvador - BA, Brazil, pp. 14771482.

Gomes da Silva Jr., J. M., Paim, C. and Castelan, E. B. (2001). Stability and stabilization of linear discretetime subject to control saturation, 1st IFAC Symp. on System Structure and Control (SSSC'01), Prague.

Gomes da Silva Jr., J. M., Reginatto, R. and Tarbouriech, S. (2004). Anti-windup design with guaranteed regions of stability for discrete-time linear systems with saturating controls, Revista Controle e Automação 14(1): 3-9.

Gomes da Silva Jr., J. M. and Tarbouriech, S. (2005). Antiwindup design with guaranteed regions of stability: an lmi-based approach, Automatica 50(1): 106-111.

Hu, T. and Lin, Z. (2003). Composite quadratic lyapunov functions for constrained control systems, IEEE Transactions on Automatic Control 48(3): 440-450.

Hu, T., Lin, Z. and Chen, B. M. (2002). An analysis and design method for linear systems subject to actuator saturation and disturbance, Automatica 38: 351-359.

Khalil, H. K. (2002). Nonlinear Systems - Third Edition, Prentice Hall.

Kokotovic, P. and Arcak, M. (2001). Constructive nonlinear control: a historical perspective, Automatica 37(5): 637-662.

Milani, B. E. A. and Coelho, A. D. (2002). Funções de lyapunov lineares por partes para sistemas lineares com controles saturáveis, Revista Controle e Automação 13(1): 42-50.

Montagner, V. F., Peres, P. L. D., Queinnec, I. and Tarbouriech, S. (2006). Determinação de regiões de estabilidade robusta para sistemas lineares incertos com saturação de atuadores: uma abordagem lmi, XVI Brazilian Automatic Control Conference, Salvador - BA, Brazil, pp. 1207-1212.

Montagner, V. F., Peres, P. L. D., Tarbouriech, S. and Queinnec, I. (2006). Improved estimation of stability regions for uncertain linear systems with saturating actuators: an LMI-based approach, 45th IEEE Conf. on Decision and Control, San Diego, CA, pp. 5429-5434. 
Tarbouriech, S., Garcia, G. and Langouet, P. (2004). Antiwindup strategy with guaranteed stability for linear systems with amplitude and dynamics restricted actuator, 6th IFAC Symp. on Nonlinear Control Systems (NOLCOSO4), Stuttgart (Germany). 